\title{
LA SUPREMA CORTE DE JUSTIGIA DE LA NAGIÓN Y LA IMPOSICIÓN DE MEDIDAS PRELIMINARES A LOS PROVEEDORES DE SERVICIOS DE INTERNET
}

\author{
Roberto GARZA BARBOSA*
}

SUMARIO: I. Introducción. II. Las medidas provisionales y el derecho internacional. III. Twentieth Century Fox Film Co. et al. vs. British Telecommunications PLC. IV. Procedimiento de infracción administrativa en materia de comercio ante el IMPI. V. Resolución definitiva del procedimiento de infracción administrativa en materia de comercio. VI. El litigio ante la Suprema Corte: Amparo en Revisión 553/2014. VII. Conclusiones.

\section{INTRODUCGIÓN}

El presente ensayo aborda un litigio por infracción a los derechos de autor y derechos conexos dentro del contexto jurídico mexicano. Ciertamente, la característica del litigio que aquí se presenta tiene una extraordinaria semejanza con el caso presentado ante la Corte Superior de Justicia, División Cancillería del Reino Unido, Twentieth Century Fox Film Co. et al. vs. British Telecommunications PLC. ${ }^{1}$ Lo anterior, debido a que en ambos litigios se emitieron medidas preliminares dirigidas a un proveedor de servicios de Internet a fin de evitar que sus usuarios tuvieran acceso a una página de Internet cuyo contenido se alegaba infractor de derechos de autor y derechos conexos.

Este litigio, además, es de gran interés, debido a que del mismo derivó el Amparo en Revisión 553/2014, resuelto el 11 de febrero de 2015 por la Segunda Sala de la Suprema Corte de Justicia de la Nación. En dicha resolución, la Segunda Sala sostuvo la constitucionalidad, tanto del artículo 199

* Profesor en el Departamento de Derecho y editor de la Revista Iustitia del Instituto Tecnológico de Estudios Superiores de Monterrey, campus Monterrey.

1 Garza Barbosa, Roberto, "El derecho de autor, las nuevas tecnologías y el derecho comparado. Una reflexión para la legislación nacional y sus desarrollos jurisprudenciales", Boletín Mexicano de Derecho Comparado, vol. 142, 2015, pp. 41 y 66.

Boletín Mexicano de Derecho Comparado nueva serie, año XLX, núm. 148 , enero-abril de 2017, pp. 459-482.

D. R. (C) 2017. UNAM, Instituto de Investigaciones Jurídicas. 
Esta revista forma parte del acervo de la Biblioteca Jurídica Virtual del Instituto de Investigaciones Jurídicas de la UNAM

bis, fracción V, de la Ley de la Propiedad Industrial, como del penúltimo párrafo del artículo 177 del Reglamento de la Ley Federal del Derecho de Autor.

Primero comenzaré por explicar las medidas preliminares en el derecho internacional; posteriormente, abordaré el litigio llevado ante la Corte Superior de Justicia del Reino Unido. Con este referente de derecho comparado, continuaré con las resoluciones emitidas por el Instituto Mexicano de la Propiedad Industrial (IMPI), tanto las relativas a medidas preliminares como la declaración de infracción dictada después de agotado el procedimiento principal. Finalmente, analizaré la resolución de la Segunda Sala de la Suprema Corte de Justicia de la Nación.

\section{LAS MEDIDAS PROVISIONALES Y EL DERECHO INTERNACIONAL}

La aparición en nuestro sistema jurídico de medidas preliminares sin audiencia de la parte contraria en litigios relacionados con propiedad intelectual se debe principalmente a dos tratados comerciales internacionales. ${ }^{1}$ Cada uno de ellos tiene un capítulo sobre propiedad intelectual. Estamos hablando del Acuerdo por el que se Establece la Organización Mundial del Comercio (OMC, o WTO por sus siglas en inglés) y su capítulo de propiedad intelectual, denominado Acuerdo sobre los Aspectos de los Derechos de Propiedad Intelectual Relacionados con el Comercio (ADPIC, o TRIP por sus siglas en inglés). Además, el Tratado de Libre Comercio de América del Norte y su capítulo XVII, relativo a propiedad intelectual.

$\mathrm{El}$ artículo 50 del ADPIC establece que las autoridades judiciales, o incluso las administrativas, podrán emitir medidas preliminares destinadas a detener la infracción o a preservar evidencia. El segundo párrafo del artículo 50 del ADPIC establece, además, que dichas medidas podrán decretarse sin audiencia de la parte contraria cuando exista la posibilidad de que "cualquier retraso cause daño irreparable al titular de los derechos". Asimismo, el tercer párrafo del mismo precepto legal establece que la autoridad que emita la medida preliminar exigirá al solicitante las pruebas "de que razonablemente disponga", a fin de demostrar que es

1 Ilardi, Alfredo y Blakeney, Michael, International Encyclopedia of Intellectual Property Treaties, Londres, Oxford University Press, 2004, p. 181.

D. R. (C) 2017. UNAM, Instituto de Investigaciones Jurídicas,

Boletin Mexicano de Derecho Comparado, núm. 148, pp. 459-482. 
Esta revista forma parte del acervo de la Biblioteca Jurídica Virtual del Instituto de Investigaciones Jurídicas de la UNAM

titular del derecho. Además, deberá demostrar que su derecho está siendo infringido o que será "objeto inminente de infracción”. Este párrafo también establece que el solicitante deberá presentar fianza o garantía "suficiente para proteger al demandado y evitar abusos". Por su parte, el cuarto párrafo del precepto legal que se analiza establece que si la medida preliminar fue dictada sin la audiencia de la parte contraria, la misma se notificará a la parte en contra de quien se pide, inmediatamente después de haberla puesto en efecto. De igual manera, se establece que el demandado podrá solicitar la revisión de dicha medida, respetando así su derecho de audiencia; todo ello para determinar si la medida se revoca o se confirma. El sexto párrafo del mismo artículo 50 del ADPIC establece que la medida preliminar será revocada en caso de que el solicitante de la misma no inicie el procedimiento que resuelva la sustancia del litigio en un tiempo razonable. Finalmente, el séptimo párrafo dispone que si la medida preliminar se revoca por causa del solicitante, o se demuestra que no hubo infracción alguna, entonces el solicitante indemnizará por daños y perjuicios al demandado.

Por su parte, el artículo 1716 del capítulo XVII del TLCAN establece medidas preliminares en términos más o menos literales al artículo 50 del ADPIC. Es decir, un precepto legal es casi literal al otro.

\section{TWENTIETH CENTURY FOX FILM CO. ET AL. VS. BRITISH TELECOMMUNICATIONS PLC}

Este litigio se le conoce como Newzbin 2. Lo anterior, debido a que el mismo parece ser la continuación de otro litigio llevado a cabo anteriormente en el mismo país. ${ }^{2}$ En ese primer litigio, se condenó al demandado Newzbin Ltd debido a que facilitaba, a través de grupos de discusión, que sus usuarios pusieran a disposición de otros usuarios obras protegidas. Si bien el demandado no cometía directamente infracción alguna, fue condenado con base en un tipo de responsabilidad solidaria establecida por la sección 16 del Copyright, Designs and Patents Act del Reino Unido, debido a que el tribunal determinó que era responsable de autorizar y procurar la infracción de obras protegidas. Consecuentemente, se le condenó a resarcir daños y

2 Twentieth Century Fox Film Corp et al. v. Newzbin Ltd, 2010 EWHC 608 (Ch), (29 de marzo de 2010). También cfr. Garza Barbosa, Roberto, cit. nota 1, p. 64.

D. R. (C) 2017. UNAM, Instituto de Investigaciones Jurídicas, Boletín Mexicano de Derecho Comparado, núm. 148, pp. 459-482. 
Esta revista forma parte del acervo de la Biblioteca Jurídica Virtual del Instituto de Investigaciones Jurídicas de la UNAM

perjuicios, y se le ordenó en definitiva a no facilitar la infracción de obras protegidas del actor.

Después de esta condena, el demandado movió sus operaciones fuera del Reino Unido. ${ }^{3}$ Lo anterior hizo nugatoria o inservible la medida definitiva emitida en el primer litigio, ya que aunque fuera hospedada en un servidor localizado en el extranjero, la página de Internet podía accederse desde el Reino Unido. Ante esta circunstancia, el titular de las obras infringidas demandó a un tercero que no procuraba la infracción, pero que en el curso normal de sus operaciones facilitaba la misma, probablemente sin tener conocimiento de ello. Se trataba del proveedor de servicios de Internet más grande del Reino Unido, British Telecommunications PLC (BT de aquí en adelante). Aquí, el remedio buscado no eran daños y perjuicios, sino el bloqueo a dicha página de Internet. En caso de tener éxito, el actor repetiría la misma estrategia en contra del resto de los proveedores de servicios de Internet de ese país; de manera que los usuarios localizados en el Reino Unido no pudieran acceder a la página de Newzbin. Antes de interponer la demanda, el actor solicitó a BT el bloqueo a dicha página de Internet, pero BT se negó.

El fundamento jurídico de la orden para restringir el acceso a la página de Internet de Newzbin fue la sección 97a del Copyright, Designs and Patents Act del Reino Unido. Dicha sección contiene los requisitos específicos a fin de emitir este tipo de órdenes en contra de proveedores de servicios de Internet. El primer requisito es que el proveedor de servicios de Internet tenga conocimiento real de la infracción en cuestión. La interpretación de lo que dicho conocimiento significa puede variar enormemente; sin embargo, en este caso el Tribunal sostuvo que si bien "el abogado [de la actora] aceptó que no podría probar que BT tenía conocimiento real de la infracción en particular de cada obra protegida... también, alegó que BT tenía conocimiento general sobre usuarios y operadores de Newzbin2 usando su servicio para infringir derechos de autor...".

Derivado de lo anterior, el Tribunal sostuvo que BT tenía conocimiento general de que algunos de sus suscriptores infringían derechos a través de la página de Newzbin. Dicha conclusión derivó de las siguientes

3 Trentieth Century Fox Film Co. et al. vs. British Telecommunications PLC, 2011 EWHC 1981(Ch), 28 de julio de 2011, pfos. 2 y 6.

4 Ibidem, pfo. 157.

D. R. (C) 2017. UNAM, Instituto de Investigaciones Jurídicas,

Boletin Mexicano de Derecho Comparado, núm. 148, pp. 459-482. 
Esta revista forma parte del acervo de la Biblioteca Jurídica Virtual del Instituto de Investigaciones Jurídicas de la UNAM

deducciones: que BT tenía conocimiento de que usuarios y operadores de Newzbin infringían derechos de autor a gran escala; y tenía conocimiento de que usuarios de Newzbin incluían a suscriptores de BT. Con ello, dio por satisfecho el requisito de conocimiento requerido por la sección 97a del Copyright, Designs and Patents Act del Reino Unido a fin de emitir la orden para restringir el acceso a la página de Internet de Newzbin.

Dentro del litigio, BT argumentó que la orden de restricción violaba el artículo 15(1) de la Directiva sobre Comercio Electrónico, que prohíbe obligar a proveedores de servicios de Internet a monitorear información que se transmite a través de sus servicios. Sin embargo, el Tribunal sostuvo que la orden no se trataba de una orden de monitorear información que pasa a través de los servidores de BT, sino de una obligación específica y limitada de restringir el acceso a un sitio de Internet determinado de manera automatizada.

Finalmente, BT argumentó que dicha orden era contraria al artículo 10 del Convenio Europeo de Derechos Humanos, que contiene el derecho de libertad de expresión, debido a que se violaría el derecho de los usuarios al acceso de la información, lo que constituye una vertiente de la libertad de expresión. En este sentido, BT alegó que el artículo 10 de Convenio Europeo de Derechos Humanos debía prevalecer sobre el artículo 97a del Copyright, Designs and Patent Act del Reino Unido. El Tribunal desestimó dicho argumento estableciendo que ningún precepto legal prevalece sobre el otro, sino que la solución provendría de un balance entre ambos derechos, el de libertad de expresión y el derecho de autor, y que en cada caso específico la proporcionalidad de valores y medidas a tomar determinaría la pertinencia de la orden de restricción.

Es importante notar que el artículo 97a del Copyright, Designs and Patent Act del Reino Unido implementa en ese país el artículo 9o. de la Directiva Europea 2004/48/CE Relativa a las Medidas y Procedimientos Destinados a Garantizar el Respeto de los Derechos de Propiedad Intelectual. A su vez, esta directiva armoniza la implementación del capítulo de observancia y consecución de derechos del ADPIC dentro de la Unión Europea. ${ }^{5}$ Es decir, los preceptos jurídicos fundamento de las medidas

5 Boardman, Michael, "Digital Copyright Protection and Graduated Response: A Global Perspective", Loyola of Los Angeles International \& Comparative Law Review, vol. 33, 2011 , pp. 223 y 227. 
Esta revista forma parte del acervo de la Biblioteca Jurídica Virtual del Instituto de Investigaciones Jurídicas de la UNAM

preliminares, tanto en el litigio llevado a cabo en el Reino Unido como en el litigio mexicano que nos ocupa (artículos 199 bis y 199 bis 1 de la Ley de la Propiedad Industrial), representan la implementación en sus respectivos sistemas jurídicos del artículo 50 del ADPIC. La única diferencia consiste en que la sección 97 a está dirigida específicamente a proveedores de servicios de Internet, en tanto que las provisiones en la Ley de la Propiedad Industrial se encaminan a cualquier persona, sea o no proveedor de servicios de Internet. Además, como lo mostraré más delante, en los preceptos legales mexicanos no se requiere que la persona en contra de quien se emite una medida preliminar tenga conocimiento alguno de la infracción.

\section{PROGEDIMIENTO DE INFRACGIÓN ADMINISTRATIVA EN MATERIA DE COMERGIO ANTE EL IMPI}

\section{Resoluciones del IMPI en las que ordena la imposición de medidas preliminares}

En el procedimiento encontramos varios oficios en los que se ordenó la imposición de medidas preliminares, todos ellos emitidos el 27 de noviembre de 2013. Una de estas resoluciones fue dirigida al presunto infractor, ${ }^{6}$ y el resto lo fueron a diversos proveedores de servicios de Internet, respectivamente. ${ }^{7}$ Es importante mencionar que estos últimos eran terceras partes dentro del litigio.

Tanto la orden dirigida al presunto infractor como las órdenes dirigidas a los proveedores de servicios de Internet fueron fundamentadas en los mismos preceptos legales. A continuación explicaré los más importantes.

6 Instituto Mexicano de la Propiedad Intelectual, Subdirección Divisional de Infracciones Administrativas en Materia de Comercio, Sociedad de Autores y Compositores de México, S. de G. C. de I. P. y otros vs. Daniel Cuellar González y o Daniel González Cuéllary /o Titular, Propietario o Responsable del Sitio Web WWW.BA-K.COM, expediente I. M. C. 2036/2013 (M-340) 20995, Resolución folio: 39632, del 27 de noviembre de 2013, en http:/vidoc.impi.gob.mx/ (visitado el 7 de diciembre de 2015).

7 Ibidem, Resoluciones del folio 39633 al folio 39640, todas ellas del 27 de noviembre de 2013, dirigidas respectivamente a: Teléfonos de México, S. A. B. de C. V.; Cablevisión S. A. B. de C. V.; Cablemas Telecomunicaciones, S. A. de C. V.; Mega Cable, S. A. de C. V.; Avantel, S. de R. L. de C. V.; Axtel, S. A. B. de C. V.; Alestra, S. de R. L. de C. V., y Iusacell PCS de México, S. A. de C. V.

D. R. (C) 2017. UNAM, Instituto de Investigaciones Jurídicas,

Boletin Mexicano de Derecho Comparado, núm. 148, pp. 459-482. 
Esta revista forma parte del acervo de la Biblioteca Jurídica Virtual del Instituto de Investigaciones Jurídicas de la UNAM

El artículo 231 de la Ley Federal del Derecho de Autor establece las infracciones a los derechos de autor en materia de comercio. El carácter de las infracciones establecidas en este precepto legal es administrativo. Cada fracción contiene un supuesto jurídico o una causa de la acción. No obstante, en su preámbulo, dicho dispositivo jurídico establece como requisito para el perfeccionamiento de cada supuesto, que la infracción se haya realizado "con fines de lucro directo o indirecto". Dado su carácter administrativo, cualquier acción que se fundamente en el artículo 231 de la legislación en estudio tendrá que comenzar en el IMPI, quien no está facultado para emitir condenas resarcitorias de daños y perjuicios. ${ }^{8}$ Sin embargo, antes de demandar ante un juez ordinario o federal en busca de daños y perjuicios, es necesaria la declaración de infracción decretada por el IMPI. Ahora bien, es posible demandar judicialmente sin necesidad de declaración previa, y sin tener como presupuesto de la acción los fines de lucro; pero si ese fuera el caso, la causa de la acción tendría que fundamentarse, ya sea en el artículo 21 o en el artículo 27 de la Ley Federal del Derecho de Autor. ${ }^{9}$ Empero, el presente litigio se fundamenta en las fracciones I, III y $\mathrm{X}$ del artículo 231 del mismo ordenamiento jurídico.

Por su parte, el artículo 234 de la Ley Federal del Derecho de Autor faculta al IMPI para decretar las infracciones en materia de comercio contenidas en el artículo 231 del mismo ordenamiento, conforme a los títulos sexto y séptimo de la Ley de la Propiedad Industrial, facultando además al IMPI para emitir las medidas preliminares, así como realizar las visitas de inspección establecidas en la Ley de la Propiedad Industrial.

Pasemos ahora al análisis de otro fundamento jurídico de los oficios de medidas preliminares. Se trata de los párrafos primero y segundo del artículo 177 del Reglamento de la Ley Federal del Derecho de Autor. Dicho precepto legal regula medidas preliminares, llamándolas "orden se suspensión". Específicamente establece que la medida preliminar u orden de suspensión podrá recaer sobre la transmisión, radiodifusión o comunicación al público realizadas mediante las redes de telecomunicaciones. El precepto es redundante, ya que aun en su ausencia, lo anterior se deriva de los artículos 199 bis y 199 bis 1 de la Ley de la Propiedad Industrial,

8 Ley Federal del Derecho de Autor, publicada en el Diario Oficial de la Federación el 24 de diciembre de 1996, arts. 232 y 234.

9 Tesis: 1a. XXIX/2011, Semanario fudicial de la Federación y Su Gaceta, novena época, t. XXXIII, febrero de 2011, p. 613.

D. R. (C) 2017. UNAM, Instituto de Investigaciones Jurídicas, Boletín Mexicano de Derecho Comparado, núm. 148, pp. 459-482. 
Esta revista forma parte del acervo de la Biblioteca Jurídica Virtual del Instituto de Investigaciones Jurídicas de la UNAM www.juridicas.unam.mx

cubriendo así cualquier tipo de transmisión, incluyendo las realizadas a través de Internet. Estos últimos preceptos legales pertenecen al título sexto de la Ley de la Propiedad Industrial.

Aunque de inicio la resolución también se fundamentó en la fracción IV del artículo 199 bis de la Ley de la Propiedad Industrial, la verdad es que el fundamento fue la fracción $\mathrm{V}$ del mismo precepto jurídico, ya que la fracción IV no tiene relevancia para este caso, pues regula el aseguramiento de bienes, en tanto que la $\mathrm{V}$ regula la "suspensión o cese de actos..." infractores.

En este caso, no se trata de asegurar bienes, sino más bien de ordenar la suspensión o el cese de la transmisión a través de una página de Internet. Posteriormente, en cada una de las resoluciones en donde se ordenó la imposición de medidas preliminares, el IMPI citó textualmente la fracción $\mathrm{V}$ del precepto legal en comento, en lugar de la fracción IV.

El artículo 199 bis 1 de la Ley de la Propiedad Industrial es el punto central de la resolución de medidas preliminares que nos ocupa, ya que el mismo señala con precisión los aspectos que el actor tendrá que demostrar, además de señalar los requisitos con los que tendrá que cumplir a fin de obtener dicha medida.

De esto se trata cada uno de los oficios que ordenan las medidas preliminares. Primeramente, comienzan estableciendo cómo cada uno de los actores demostró la titularidad del derecho. Así, por ejemplo, Armando Manzanero Canche, representado por la Sociedad de Autores y Compositores de México, S. de G. C. de I. P. acreditó ser titular de los derechos de autor de varias obras musicales, entre ellas: "Cuando hay amor", "Adoro", "No sé tú", presentando varios discos compactos que contenían fonogramas de dichas obras musicales. Estos discos compactos se titulaban: "Las mujeres de Armando Manzanero", y "Simplemente Armando Manzanero". El IMPI dio valor probatorio a dichas pruebas fundamentándose en los artículos 197 y 210-A del Código Federal de Procedimientos Civiles, el primer precepto legal da al juzgador amplia libertad para el análisis y valoración de las pruebas, en tanto que el segundo reconoce como pruebas los medios tecnológicos, como los discos compactos presentados en este caso.

Asimismo, entre los actores tenemos a Warner Music México, S. A. de C. V., quien demostró, mediante la presentación de un disco compacto, ser el productor del fonograma denominado "Sueños Líquidos". Junto a 
Esta revista forma parte del acervo de la Biblioteca Jurídica Virtual del Instituto de Investigaciones Jurídicas de la UNAM

dicha prueba, también presentó una copia certificada del certificado de inscripción de dicho fonograma ante el Instituto Nacional del Derecho de Autor (Indautor).

También figuraban como actores, titulares de derechos extranjeros, como Warner Bros Entertainment, Inc., quien demostró ser el productor de la obra cinematográfica denominada "El Último Samurai", mediante la exhibición del soporte material de dicha obra. La misma fue cotejada en la misma diligencia a la que me he referido en anteriores líneas, del 11 de noviembre de 2013. Al igual que el resto de las pruebas consistentes en soportes materiales electrónicos o discos compactos, las mismas fueron valoradas y admitidas de conformidad con los artículos 197 y 210-A del Código Federal de Procedimientos Civiles.

Después de demostrar la existencia del derecho, así como su titularidad, el segundo punto a demostrar de acuerdo con los propios oficios fue "la posible violación a los derechos, así como la posibilidad de sufrir un daño irreparable". Lo anterior, de acuerdo con lo preceptuado por la fracción I del artículo 199 bis 1de la Ley de la Propiedad Industrial. Este punto quedó demostrado, a juicio del IMPI, mediante la diligencia de inspección ocular llevada a cabo el 11 de noviembre de 2013, en la que se demostró que la página de internet http://www.ba-k.com, con dirección IP 188.165.12.184 contenía "ligas o vínculos, que a su vez permiten la reproducción de dichos derechos protegidos, lo que evidencia la presunta violación a cada uno de los mencionados derechos". De dicha diligencia se levantó un acta circunstanciada, a la que el IMPI le dio valor probatorio, de acuerdo con los artículos 129, 130, 197 y 202 del Código Federal de Procedimientos Civiles.

Los actores adjuntaron un billete de depósito expedido por el Banco del Ahorro Nacional y Servicios Financieros por la cantidad de diez mil pesos, a fin de garantizar posibles daños y perjuicios que se pudieran ocasionar a la parte demandada. Aunque no lo señala expresamente en ese párrafo de la resolución, el fundamento de lo anterior es la fracción II del artículo 199 bis 1 de la Ley de la Propiedad Industrial. En cada uno de los oficios emitidos que otorgan la medida preliminar, el IMPI consideró la gravedad de la infracción, así como la fianza otorgada. 
Esta revista forma parte del acervo de la Biblioteca Jurídica Virtual del Instituto de Investigaciones Jurídicas de la UNAM

\section{RESOLUGIÓN DEFINITIVA DEL PROCEDIMIENTO DE INFRACGIÓN} ADMINISTRATIVA EN MATERIA DE COMERGIO

La resolución definitiva fechada el 28 de noviembre de 2014 comenzó dando cuenta de los antecedentes del expediente, es decir, los hechos y actos procesales, por llamarlos de alguna manera. ${ }^{10}$ Dentro de lo más relevante del procedimiento tenemos que todos los actores, a través de sus diversos representantes, comparecieron mediante escrito presentado el 15 de octubre de 2013, a fin de solicitar la declaración de infracción. Asimismo, la resolución da cuenta de la imposición de las medidas preliminares descritas en el apartado anterior, así como de las manifestaciones que el demandado hizo a dichas medidas preliminares. Además, encontramos que el demandado presentó su escrito de contestación a la solicitud de infracción en materia del comercio el 2 de mayo de 2014. En dicho escrito, el demandado, además de dar contestación a la acción de infracción, solicitó la nulidad de diversas actuaciones dentro del procedimiento. El 26 de ese mismo mes, los actores presentaron un escrito en el que expresaron las manifestaciones correspondientes al escrito de contestación presentado por el demandado. Estos últimos escritos se presentaron a través de "transmisión telefónica facsimilar".

La inspección ocular ofrecida por el demandado en sus manifestaciones a las medidas preliminares se desahogó el 26 de junio de 2014, en tanto que la inspección ocular ofrecida por los actores sobre la página de Internet wrweba-k.com se desahogó el 7 de agosto de 2014. Finalmente, el expediente se puso en estado de alegatos, mismos que fueron desahogados por ambas partes, y así, agotado el procedimiento, se procedió al dictado de la resolución que nos ocupa.

En su parte considerativa, después de fundamentar su competencia y antes de entrar al estudio de la acción, el IMPI dio cuenta de las pruebas ofrecidas, básicamente las mismas ofrecidas en lo que denominó "expediente de medidas preliminares". Luego, estudió la personalidad de cada

10 Instituto Mexicano de la Propiedad Intelectual, Subdirección Divisional de Infracciones Administrativas en Materia de Comercio, Sociedad de Autores y Compositores de México, S. de G. C. de I. P. y otros vs. Daniel Cuellar González y /o Daniel González Cuellar y /o Titular, Propietario o Responsable del Sitio Web WWW.BA-K.COM, Expediente I.M.C. 2036/2013 (M-340) 20995, Resolución folio: 39832, de fecha 28 de noviembre de 2014, en <http://vidocimpi.gob.mx/> (visitado el 7 de diciembre de 2015), p. 1.

D. R. (C) 2017. UNAM, Instituto de Investigaciones Jurídicas, Boletin Mexicano de Derecho Comparado, núm. 148, pp. 459-482. 
Esta revista forma parte del acervo de la Biblioteca Jurídica Virtual del Instituto de Investigaciones Jurídicas de la UNAM

una de las partes para posteriormente declarar inoperantes tres incidentes de nulidad de actuaciones, el que referimos anteriormente y otros dos presentados con posterioridad. Es decir, después del primer incidente, la parte demandada presentó otro incidente de nulidad, esta vez en contra de la audiencia llevada a cabo el 7 de agosto de 2014. El mismo también fue declarado inoperante por la autoridad. Los argumentos vertidos en el primero de los incidentes fueron varios: desde el hecho de que en la audiencia de visita ocular a fin de emitir las medidas preliminares se creó un usuario y una contraseña para acceder a la página, hasta aquel en el que el demandado aseguró que en la visita de inspección no se descargaron todos y cada uno de los archivos en los que se alegaba infracción. El demandado también se dolía de no haber sido notificado para tal audiencia.

La acción de infracción administrativa en materia del comercio estaba basada en tres supuestos contenidos en el artículo 231 de la Ley Federal del Derecho de Autor, que el IMPI transcribió en su resolución:

Constituyen infracciones en materia del comercio las siguientes conductas cuando sean realizadas con fines de lucro directo o indirecto: I. Comunicar o utilizar públicamente una obra protegida por cualquier medio, y de cualquier forma...; III. Producir, reproducir, almacenar, distribuir, transportar o comercializar copias de obras, fonogramas, videogramas...; X. Las demás infracciones a las disposiciones de la Ley...

El IMPI denominó a cada una de estos supuestos o causas de acción "causal de infracción". Al comenzar el análisis de cada una de ellas, enumeró los elementos para su actualización. Así, para la causa de la acción establecida por la fracción I del artículo 231 de la Ley Federal del Derecho de Autor, el IMPI enumeró tres elementos a demostrar: $a$ ) que el solicitante sea el titular de la obra protegida "por los derechos de autor"; $b$ ) "que se comunique o utilice públicamente una obra por cualquier medio y de cualquier forma, sin la autorización previa y expresa de su respectivo titular", y c) el lucro directo o indirecto. ${ }^{11}$

$\mathrm{Al}$ iniciar el análisis del primer elemento, el IMPI sostuvo que tres de los actores no acreditaron ser titulares de derechos de autor, debido a que comparecieron en su carácter de titulares de derechos conexos, por lo que el supuesto normativo establecido por la fracción I del artículo 231 de la Ley

11 Ibidem, p. 22.

D. R. C 2017. UNAM, Instituto de Investigaciones Jurídicas, Boletín Mexicano de Derecho Comparado, núm. 148, pp. 459-482. 
Esta revista forma parte del acervo de la Biblioteca Jurídica Virtual del Instituto de Investigaciones Jurídicas de la UNAM

Federal del Derecho de Autor no se perfeccionó en su caso, debido a que dicho supuesto favorece a titulares de derechos de autor, no abarcando así derechos conexos. ${ }^{12}$ Resulta importante mencionar que no es lo mismo ser el autor de la letra de la música, protegida por derechos de autor, a ser el productor del fonograma en donde se interpreta dicha letra, en cuyo caso la protección es mediante los derechos conexos. El IMPI sostuvo que para estos actores resultaría innecesario analizar los otros dos elementos de esta causa de la acción.

Por su parte, el actor, Armando Manzanero Canche, presentó como prueba para demostrar la titularidad del derecho de autor sobre la letra varias canciones, dos discos compactos. El primer disco compacto con el título "Las mujeres de Manzanero. Mis nuevos boleros", contenía trece canciones, que fueron reproducidas en la audiencia de pruebas llevada a tal efecto y transcrita la letra de cada una de ellas. El segundo disco compacto titulado "Simplemente Armando Manzanero. Edición de lujo", contenía quince canciones, mismas que fueron reproducidas y transcritas las letras de la misma manera que el disco anterior. A estos discos compactos les fue otorgado valor probatorio, de conformidad con los artículos 197 y 210-A del Código Federal de Procedimientos Civiles, ya que si bien no son documentales públicas, los mismos no fueron objetados por la contraparte. De manera que, de acuerdo con el IMPI, el actor acreditó la fijación de su obra y el reconocimiento de la misma, de acuerdo con los artículos 3o., 5o. y 6o. de la Ley Federal del Derecho de Autor, que establecen que para que la obra sea reconocida debe ser original y estar fijada, definiendo además, la fijación. Por lo tanto, con relación al actor Armando Manzanero Canche, el IMPI determinó por acreditado el primer supuesto de la causal de infracción en análisis.

Con relación al actor de la demanda, Reyli Barba Arrocha, el análisis fue básicamente el mismo que el realizado para el actor Armando Manzanero Canche, ya que las pruebas presentadas fueron dos discos compactos y un disco DVD. En relación con la mayoría de las canciones, se tuvo por acreditado el derecho, así como la titularidad del mismo, de conformidad con los mismos preceptos legales aplicables al actor anterior. Sin embargo, en relación con ciertos títulos de los que era autor de la música y coautor de

12 Idem. Dichos actores eran: Sony Bmg Entertainment de México, S. A. de G. V., Warner Music de México, S. A. de C. V., Warner Bros. Entertainment, Inc, y Universal City Studios LLP.

D. R. (C) 2017. UNAM, Instituto de Investigaciones Jurídicas,

Boletin Mexicano de Derecho Comparado, núm. 148, pp. 459-482. 
Esta revista forma parte del acervo de la Biblioteca Jurídica Virtual del Instituto de Investigaciones Jurídicas de la UNAM

la letra, no se tuvo por acreditada la personalidad, toda vez que de acuerdo con los artículos 80 y 81 de la Ley Federal del Derecho de Autor, el actor de la demanda tuvo que dar aviso a su coautor de la acción por intentar, cuestión que no se desprendió del expediente. Consecuentemente, sólo se le tuvo por acreditando la titularidad del derecho de las canciones en las que aparecía como autor de la letra, así como de la música.

Con relación al segundo elemento de esta causal de infracción, es decir, que el demandado haya "comunicado o utilizado públicamente las obras... protegidas por cualquier medio y de cualquier forma", ${ }^{13} \sin$ autorización previa y expresa del titular de cada obra, el IMPI comenzó citando la definición del término "comunicación pública", contenida en la fracción III del artículo 16 de la Ley Federal del Derecho de Autor. Después de notar que no hay definición de "utilizar" en la ley en comento y tampoco en los ordenamientos de aplicación supletoria, el IMPI citó las definiciones de "utilizar", "usar", y "aprovechar" del Diccionario de la lengua española. Lo mismo ocurrió con el término "difusión", para cuyo caso el IMPI citó el Glosario de términos más usuales en derecho de autor, publicado por el Indautor. De todas estas definiciones, el IMPI concluyó que la utilización pública tiene un alcance más amplio que la comunicación pública, debido a que incluye "cualquier forma de aprovecharse de la obra", poniéndola a disposición del público por cualquier medio.

Posteriormente, la resolución da cuenta de las pruebas presentadas a fin de demostrar el segundo elemento de esta causal de infracción: en primer término, la parte actora presentó una documental pública consistente en una fe de hechos notarial sobre el sitio de Internet objeto de la controversia (http://wwre.ba-k.com), a la que el IMPI dio valor probatorio pleno. De acuerdo con la propia resolución, en dicho instrumento notarial se dio cuenta del contenido del sitio, en el que se encontraron diversas obras protegidas, entre ellas las de los actores de la demanda. Asimismo, se dio cuenta de las impresiones, así como de las grabaciones en formato electrónico obtenidas del sitio en cuestión durante la mencionada diligencia notarial. Estas impresiones y grabaciones fueron anexadas como apéndices del instrumento notarial.

Además, la parte actora también presentó como prueba la documental pública, que hizo consistir en el acta levantada con motivo de la ins-

\footnotetext{
13 Ibidem, p. 90.
} 
Esta revista forma parte del acervo de la Biblioteca Jurídica Virtual del Instituto de Investigaciones Jurídicas de la UNAM

pección ocular llevada a cabo el 11 de noviembre de 2013 por el propio IMPI. Todo ello, con objeto de inspeccionar los soportes materiales presentados como prueba por la parte actora para demostrar la titularidad de los derechos, así como inspeccionar el contenido de la página de Internet objeto de la controversia. Se presentaron además otras pruebas, como la cédula de notificación de las medidas preliminares, y documentales privadas que contenían impresiones de los contenidos de la página de Internet objeto de la controversia, así como una documental pública consistente en la inspección ocular levantada sobre la página de Internet denominada "Facebook de la demandada".

En la parte medular de su razonamiento, el IMPI estableció que los actores acreditaron su interés jurídico, y que de las mismas pruebas se demostró que la página de Internet administrada por el demandado estaba llevando a cabo

...actos mediante los cuales las obras musicales base de la acción se ponen al alcance general, es decir, cualquier persona puede ingresar a la página www.ba-k.com, y mediante una búsqueda localizar cualquiera de las canciones... y como resultado de esa búsqueda obtener varios links que al darles click hace accesible... el ingreso a diversas páginas en las cuales se encuentran copias digitales de dichas obras mismas que pueden ser escuchadas y descargadas... ${ }^{14}$

El anterior párrafo da cuenta de un hecho que más adelante es analizado por la autoridad. Consiste en que la página infractora no infringe directamente los derechos, sino que da acceso a páginas de terceros, en donde se comete directamente la infracción. Es decir, aunque no se expresa literalmente, estamos frente a un caso de responsabilidad solidaria. Anteriormente he abordado este tema desde una perspectiva de derecho comparado, y he argumentado que dicha responsabilidad podría existir en nuestro sistema jurídico. Sin embargo, la línea de argumentación que abordé en aquella ocasión se relaciona con una causa de infracción basada en el artículo 27 de la Ley Federal del Derecho de Autor y no en la causa de la acción del presente caso, basada en artículo 231 del mismo ordenamiento. ${ }^{15}$

14 Ibidem, p. 133.

15 Garza Barbosa, Roberto, "El derecho de autor, las nuevas tecnologías y el derecho comparado. Una reflexión para la legislación nacional y sus desarrollos jurisprudenciales", 
Esta revista forma parte del acervo de la Biblioteca Jurídica Virtual del Instituto de Investigaciones Jurídicas de la UNAM

Sin embargo, de acuerdo con la resolución, la conducta del demandado encuadra directamente en el supuesto normativo. Es decir, no hay responsabilidad solidaria, sino que el propio acto de comunicar públicamente o utilizar la obra en cuestión entra en el supuesto normativo establecido en la fracción I del artículo 231 de la Ley Federal del Derecho de Autor. Sin embargo, el IMPI también fundamentó su resolución en el propio artículo 27 del mismo ordenamiento, así como en el artículo 8 del Tratado de la OMPI sobre Derecho de Autor. ${ }^{16}$ Lo anterior, debido al amplio alcance dado a la fracción I del artículo 231 de la ley que nos ocupa. Dicha interpretación proviene de las definiciones mencionadas anteriormente. Así, el IMPI sostuvo que "aquél sujeto que proporcione la mera posibilidad de acceso directo a una obra mediante los hipervínculos... conducta que constituye una comunicación pública en la modalidad de puesta a disposición, vulnerará los derechos patrimoniales de comunicación pública de la obra". ${ }^{17}$

El tercer elemento de esta causa de la acción, es decir, el lucro directo o indirecto, quedó debidamente probado con las pruebas ya mencionadas, ya que de la fe notarial, así como de la inspección ocular, se desprendió que la página objeto de la controversia contenía diversos anuncios publicitarios. Por lo tanto, el IMPI asumió el beneficio económico debido al costo que dicha publicidad representaría para las empresas anunciantes. Consecuentemente, el IMPI declaró la infracción de la fracción I del artículo 231 de la Ley Federal del Derecho de Autor.

La segunda causa de infracción, basada en la fracción III del artículo 231 de la legislación de la materia, define como conductas infractoras "producir, reproducir, almacenar, distribuir..." obras protegidas, pero no solamente por derechos de autor, sino también por derechos conexos, como los que poseen los productores de fonogramas, así como artistas, in-

Boletín Mexicano de Derecho Comparado, vol. 142, 2015, pp. 41 y 79-81. El fundamento sería el propio artículo 27 de la Ley Federal del Derecho de Autor, en su párrafo inicial, debido a la frase "autorizar o prohibir", o bien el artículo 1917 del Código Civil Federal.

16 Que establece el derecho de puesta a disposición (on demand availability right), que es un subgénero del derecho de comunicación, pero está diseñado para cubrir la puesta a disposición asincrónica en la que no es necesario probar la recepción, es decir, aquella que ocurre a través del Internet. Cfr. Garza Barbosa, Roberto, Derechos de autor y derechos conexos. Marco jurídico internacional, México, Porrúa, 2009, p. 58.

17 IMPI, Resolución folio: 39832, del 28 de noviembre de 2014, véase nota 11, p. 134. 
Esta revista forma parte del acervo de la Biblioteca Jurídica Virtual del Instituto de Investigaciones Jurídicas de la UNAM

térpretes o ejecutantes. Por lo tanto, esta causa de acción tiene un espectro más amplio que la primera causa de infracción analizada. Es decir, aquí no solamente se protege al autor de la letra o la música, sino también al productor de fonogramas, como es el caso de Sony Music de Entertainment de México, S. A. de C. V., o bien de Warner Music de México, S. A. de C. V., quienes son productores de fonogramas y no fueron cubiertos por la anterior causa de la acción. Además, cubre a los actores de la demanda, en su carácter de intérpretes de las canciones de las que son titulares como autores o compositores. Es decir, son compositores e intérpretes de las canciones objeto del litigio.

Dos de los tres elementos a demostrar son muy parecidos a la primera causa de la acción, y solamente cambia el elemento descrito literalmente en la propia fracción III del artículo 231 del mismo ordenamiento jurídico. Es decir, para todas las causas de la acción contenidas en el artículo 231 de la Ley Federal del Derecho de Autor se debe demostrar que se es titular del derecho de autor o derecho conexo, además de demostrar el lucro directo o indirecto. La causa de acción específica refiere a las ya mencionadas conductas definidas por la fracción III: "producir, reproducir, almacenar, distribuir...".

Aquí el IMPI se centró en volver a enumerar las pruebas, que básicamente fueron las mismas analizadas en la anterior causa de infracción. Además, el IMPI analizó el elemento que es diferente, que está propiamente definido en la fracción III, para lo cual empleó diversas definiciones de casi todos los términos enumerados por dicha fracción, como las establecidas en la propia Ley Federal del Derecho de Autor, en el Glosario de términos publicado por el Indautor, así como en el propio Diccionario de la lengua española. Asimismo, citó el artículo 14 del Tratado de la OMPI sobre Interpretación o Ejecución y Fonogramas, que es un espejo del artículo 8o. del Tratado de la OMPI sobre Derecho de Autor, conteniendo el derecho de puesta a disposición (on demand availability right).

Posteriormente, el IMPI concluyó que de las pruebas aportadas, los actores demostraron el elemento de distribución, debido a los links que remitían a los usuarios de la página de Internet del demandado hacia páginas de terceros, que contenían las obras objeto de la controversia. Asimismo, sostuvo que del expediente no se desprendió autorización alguna por parte de los actores a los demandados para efectuar dicha distribución. Por lo tanto, el IMPI sostuvo que al quedar probada la distribución, 
Esta revista forma parte del acervo de la Biblioteca Jurídica Virtual del Instituto de Investigaciones Jurídicas de la UNAM

se perfeccionó el elemento normativo de la fracción III del artículo 231 de la legislación en comento. Respecto de las demás hipótesis contenidas en el mismo precepto legal de producción, almacenamiento, transportación o comercialización, el IMPI concluyó que las mismas no se actualizaron. Sin embargo, dicho precepto legal contiene varios supuestos normativos, por lo que al perfeccionarse alguno de los mismos, dígase la distribución, se perfecciona el elemento normativo para declarar la infracción.

Sobre la causal de infracción contenida en la fracción X del artículo 231, misma que es residual y establece como infracción las "demás infracciones a las disposiciones de la Ley que impliquen conducta a escala comercial...", el IMPI sostuvo que la misma no se perfeccionó, debido a que "la referida fracción debe estar forzosamente relacionada con alguna otra disposición de la Ley... y de la solicitud... no se desprende ninguna relación... con algún otro precepto legal... aplicable".

Habiendo quedado declarada la infracción de las fracciones I y III del artículo 231 de la Ley Federal del Derecho de Autor, el IMPI procedió a fundamentar la imposición de la multa para el demandado, basándose para ello en el artículo 220 del mismo ordenamiento. Finalmente, el IMPI decretó hacer definitivas de medidas provisionales impuestas, así como la devolución de la garantía exhibida por los actores al solicitar dichas medidas provisionales.

\section{El Litigio ante la Suprema Corte. Amparo EN REVISIÓN 553/2014}

\section{Hechos}

El 19 de diciembre de 2013, Alestra, S. de R. L. de C. V., promovió el juicio de amparo indirecto, alegando la inconstitucionalidad del artículo 199 bis, fracción V, de la Ley de la Propiedad Industrial. ${ }^{18}$ Asimismo, alegó la

18 Segunda Sala de la Suprema Corte de Justicia de la Nación, Amparo en Revisión $553 / 2014$, ***********, 11 de febrero de 2015. Sentencia obtenida a través del módulo de acceso a la información de la Suprema Corte de Justicia de la Nación, en http://wrww2. scjn.gob.mx/ConsultaTematica/PaginasPub/TematicaPub.aspx (visitado el 9 de mayo de 2016), pp. 1-3. Esta sentencia es una versión pública con los nombres de las partes tachados con asteriscos. Sin embargo, de la misma se desprende el número de oficio emitido por el 
Esta revista forma parte del acervo de la Biblioteca Jurídica Virtual del Instituto de Investigaciones Jurídicas de la UNAM

inconstitucionalidad del artículo 177, penúltimo párrafo, del Reglamento de la Ley Federal del Derecho de Autor. Como primer acto de aplicación, señaló la imposición de las medidas preliminares contenidas en el oficio 39639, del 27 de noviembre de 2013. Dicho oficio fue analizado en el segundo capítulo del presente ensayo. El 10 de enero de 2014, el Juzgado Decimoquinto de Distrito en Materia Administrativa en el Distrito Federal admitió la demanda y ordenó la formación del expediente. Posteriormente, celebró la audiencia constitucional, y dictó sentencia, en la que sobreseyó el juicio de amparo planteado, negando así la protección solicitada.

En contra de dicha resolución, tanto la quejosa Alestra, como el IMPI, interpusieron un recurso de revisión. Además, el Ejecutivo Federal, a través de la Secretaría de Economía, interpuso la revisión adhesiva. Después de haber admitido dichos recursos, el 6 de agosto de 2014 el Primer Tribunal Colegiado de Circuito del Centro Auxiliar de la Primera Región, con residencia en el Distrito Federal, desechó el recurso de revisión interpuesto por el IMPI, dejando a salvo la jurisdicción de la Suprema Corte de Justicia de la Nación.

\section{Análisis jurídico}

La primera consideración de la Segunda Sala de la Suprema Corte de Justicia de la Nación fue el fundamento de su propia competencia para conocer del recurso de revisión. Posteriormente, la Segunda Sala dio cuenta de los conceptos de violación planteados por la quejosa ante el juez de distrito en su demanda inicial. De ellos, los relacionados con la cuestión de constitucionalidad fueron los siguientes: que el artículo 177, penúltimo párrafo, del Reglamento de la Ley Federal del Derecho de Autor, excede la facultad reglamentaria del Ejecutivo federal, ya que otorga al IMPI facultades en materia de telecomunicaciones, que son exclusivas del Instituto Federal de Telecomunicaciones. Otro concepto de violación consistió en que el artículo 199 bis, fracción V, de la Ley de la Propiedad Industrial, genera incertidumbre jurídica, y por lo tanto es contrario al artículo 16 constitucional. Dicha incertidumbre se genera debido a que

IMPI, 39639, dirigido a la quejosa y recurrente, del 27 de noviembre de 2013. Dicho oficio de medidas cautelares fue analizado al comienzo de este estudio; el mismo fue dirigido a Alestra, S. de R. L. de C. V.

D. R. (C) 2017. UNAM, Instituto de Investigaciones Jurídicas,

Boletin Mexicano de Derecho Comparado, núm. 148, pp. 459-482. 
Esta revista forma parte del acervo de la Biblioteca Jurídica Virtual del Instituto de Investigaciones Jurídicas de la UNAM

dicho precepto jurídico remite a la propia Ley de la Propiedad Industrial en lugar de remitir a la Ley Federal del Derecho de Autor.

El tercer concepto de violación es de suma relevancia. El mismo consistió en que tanto el artículo 177, penúltimo párrafo, del Reglamento de la Ley Federal del Derecho de Autor, como el artículo 199 bis, fracción $\mathrm{V}$, violan el artículo 14 constitucional. Específicamente, la garantía de audiencia, ya que dichos preceptos legales no dan oportunidad a la persona a quien va dirigida la medida preliminar, de manifestar "lo que a su derecho corresponde y probar lo que a su interés convenga". Este concepto de violación es parecido a otro vertido posteriormente, en el que la quejosa manifestó que previamente a la emisión de medidas preliminares no se le respetó su garantía de audiencia. Es decir, estos conceptos de violación versan sobre la emisión de medidas preliminares sin audiencia de parte y la garantía de audiencia.

El juez de distrito sostuvo que la quejosa no tenía razón al afirmar que el artículo 199 bis, fracción V, de la Ley de la Propiedad Industrial, presentaba un problema en cuanto al ámbito competencial del IMPI, debido a que el legislador se encuentra facultado para ampliar, de acuerdo con determinadas circunstancias, las facultades del IMPI. En su resolución, el juez de distrito ni siquiera mencionó el artículo 177, penúltimo párrafo, del Reglamento de la Ley Federal del Derecho de Autor, debido a que el juez de distrito consideró que fue "el legislador, y no así el propio titular del Ejecutivo", quien dotó de facultades al IMPI en materia de derechos de autor. Es decir, rechazó que el Ejecutivo se haya excedido en sus facultades reglamentarias, porque solamente reconoció la aplicabilidad del artículo 199 bis, fracción V, de la Ley de la Propiedad Industrial, al presente caso. Como lo mencioné en anteriores líneas, el artículo 177, penúltimo párrafo, del Reglamento de la Ley Federal del Derecho de Autor, parece redundante, ya que dicha facultad se encuentra claramente establecida en el artículo 199 bis de la Ley de la Propiedad Industrial.

Asimismo, el juez de distrito sostuvo que el artículo 199 bis, fracción V, de la Ley de la Propiedad, no genera incertidumbre jurídica a la quejosa, ya que si bien es cierto que la Ley Federal del Derecho de Autor es el ordenamiento que faculta al IMPI para sancionar infracciones en materia de comercio a los derechos de autor y derechos conexos, también lo es que dicho ordenamiento refiere a todo el marco normativo contenido en diversos títulos de la Ley de la Propiedad Industrial. 
Esta revista forma parte del acervo de la Biblioteca Jurídica Virtual del Instituto de Investigaciones Jurídicas de la UNAM

Por último, el juez de distrito sostuvo que el artículo 199 bis, fracción $\mathrm{V}$, no viola la garantía de audiencia contenida en el artículo 14 constitucional, pues la medida precautoria emitida sin audiencia de la parte contraria se encuentra inmersa en un procedimiento de origen, "lo cual se traduce en que no se priva de sus derechos al gobernado".

$\mathrm{Al}$ promover el recurso de revisión, la quejosa entonces recurrente, presentó dos agravios en cuanto al tema de constitucionalidad. El primero de ellos consistió en que el juez de distrito no analizó debidamente la cuestión de conflicto de competencia planteado como concepto de violación con relación a la facultad reglamentaria del Ejecutivo Federal y el artículo 177, párrafo penúltimo, del Reglamento de la Ley Federal del Derecho de Autor. El segundo agravio consistió en que el juez de distrito faltó al principio de exhaustividad, debido a que no analizó el concepto de violación consistente en que la medida preliminar contenida en el artículo 199 bis, fracción V, de la Ley de la Propiedad Industrial, supone "una valoración ex ante de los hechos que justifica la imposición de medidas provisionales". Es decir, se precalifican los hechos como infracciones sin estar sujetos a prueba, resultando lo anterior violatorio de los principios de "legalidad, seguridad jurídica y confianza legítima".

\section{Resolución}

En cuanto al primer agravio, la Segunda Sala determinó que el juzgador debe interpretar las normas jurídicas en el sentido en el que no se produzca contradicción con algún principio constitucional, considerando que "de ser posibles varias interpretaciones, debe preferirse aquélla que salve la aparente contradicción”. Sin embargo, refirió que el penúltimo párrafo del artículo 177 del Reglamento de la Ley Federal del Derecho de Autor "amplía" las medidas provisionales que puede decretar el IMPI, de acuerdo con los artículos 199 bis y 211 bis de la Ley de la Propiedad Industrial. Dentro de la línea de interpretación que establece que se debe interpretar el precepto jurídico de manera que no contradiga el precepto constitucional, lo más lógico hubiera sido interpretar al artículo 177 como si el mismo no ampliara las facultades otorgadas por el legislador a través de los preceptos referidos de la Ley de la Propiedad Industrial, sino más bien como si aclarara el alcance de dichos precep- 
Esta revista forma parte del acervo de la Biblioteca Jurídica Virtual del Instituto de Investigaciones Jurídicas de la UNAM

tos legales. Es decir, interpretar que el Ejecutivo no "amplió", porque no lo hizo, sino más bien aclaró el alcance de las facultades definidas por el legislador en la Ley de la Propiedad Industrial, hubiera resultado más lógico. Sin embargo, la línea de argumentación pareció romperse al establecer que dicho artículo 177 "amplía" las facultades otorgadas por los artículos 199 bis y 211 bis de la Ley de la Propiedad Industrial.

Posteriormente, la Suprema Corte de Justicia de la Nación explicó los límites de la facultad reglamentaria del Ejecutivo Federal de acuerdo con la fracción I del artículo 89 constitucional. Explicó, con una tesis emitida por la propia Segunda Sala, los principios de dicha facultad, como lo es el de reserva de ley y el principio de subordinación jerárquica. Es de llamar la atención que dentro de la citada tesis se puede leer que este último principio establece que "el ejercicio de la facultad reglamentaria no puede modificar o alterar el contenido de la ley... detallando sus hipótesis y supuestos normativos de aplicación, sin que pueda contener mayores posibilidades...".

Entonces, el sostener que el artículo 177 del Reglamento de la Ley Federal del Derecho de Autor "amplía" facultades, podría resultar violatorio del principio de subordinación jerárquica. Sin embargo, la Segunda Sala reconoció que si bien es cierto que el juez de distrito sostuvo que el artículo 177 no viola el principio de reserva de ley, también lo es que el juez no analizó el argumento de la quejosa en el que se dolía de que dicho precepto reglamentario "invade la esfera del órgano regulador en materia de telecomunicaciones". En este sentido, la Suprema Corte sostuvo que la quejosa interpretó incorrectamente el precepto legal, ya que el mismo no regula las telecomunicaciones, sino más bien la Ley Federal del Derecho de Autor, cuyo objeto es la protección de los autores, artistas, intérpretes y ejecutantes. Por lo tanto, su Reglamento puede contener la posibilidad de cesar infracciones, tales como "la representación, recitación, ejecución pública, transmisión y comunicación al público por redes de telecomunicaciones". De acuerdo con el mismo razonamiento, lo anterior "no inhibe el uso, aprovechamiento y explotación del espectro radioeléctrico, las redes públicas de telecomunicaciones... lo que es parte del objetivo que persigue la Ley Federal de Telecomunicaciones y Radiodifusión". Como lo he mencionado en anteriores líneas, el artículo 177 resulta redundante y no amplía nada de lo que establecen los artículos 199 bis y 211 bis de la Ley de la Propiedad Industrial. 
Esta revista forma parte del acervo de la Biblioteca Jurídica Virtual del Instituto de Investigaciones Jurídicas de la UNAM

Asimismo, y aunque no era parte del agravio en cuestión, la Segunda Sala sostuvo que el precepto legal en cuestión no es contrario a los artículos 6o. y 7 o. constitucionales, que contienen el derecho a la información, así como el derecho a la libertad de expresión y de información, ya que los mismos no son derechos absolutos, sino derechos con ciertas limitaciones.

En cuanto al segundo agravio, en donde la recurrente argumentaba que el juez de distrito no analizó el concepto de violación consistente en que la medida preliminar contenida en el artículo 199 bis, fracción V, de la Ley de la Propiedad Industrial, supone una valoración previa de los hechos sin audiencia de la parte afectada, violando así la garantía de audiencia contenida en el artículo 16 constitucional; la Segunda Sala sostuvo que, efectivamente, el juez de distrito "no hizo análisis alguno respecto al planteamiento..."; por lo tanto, se avocó el análisis de dicho agravio.

La Segunda Sala comenzó transcribiendo y explicando el primer párrafo del artículo 16 constitucional. Posteriormente, contextualizó el artículo 199 bis dentro del capítulo II de la Ley de la Propiedad Industrial, que regula el procedimiento de declaración administrativa. Después de transcribir los artículos que en dicho capítulo regulan las medidas preliminares, comenzó su análisis sustantivo. Lo primero que la Segunda Sala sostuvo, es que si bien las medidas se otorgan sin audiencia de la contraparte, las mismas deben contar con ciertos requisitos. Así, sostuvo que gracias a estos requisitos, que ya he analizado anteriormente, "el precepto combatido cumple con las garantías de seguridad y certeza jurídica...". Además, determinó que el IMPI puede requerir que se aumente la fianza, y que la medida preliminar no significa prejuzgar la responsabilidad del infractor, sino que la misma es provisional y que puede ser modificada. Asimismo, sostuvo que las medidas provisionales están sujetas a lo que resulte del procedimiento de infracción, por lo que se respetan el principio de seguridad jurídica y la garantía de audiencia.

Consecuentemente, la Segunda Sala de la Suprema Corte de Justicia de la Nación declaró infundados los agravios presentados, dejando a salvo la jurisdicción del Tercer Tribunal Colegiado en Materia Administrativa del Primer Circuito a fin de que se pronuncie sobre las cuestiones de legalidad esgrimidas por la quejosa en sus agravios. 
Esta revista forma parte del acervo de la Biblioteca Jurídica Virtual del Instituto de Investigaciones Jurídicas de la UNAM

\section{CONCLUSIONES}

El análisis de las sentencias desde una perspectiva de derecho comparado ofrece un invaluable laboratorio para analizar cómo se han resuelto los litigios derivados del uso de las nuevas tecnologías, ya sea como fuente de ideas para los litigantes, o bien para que el legislador contextualice determinadas figuras jurídicas del sistema jurídico mexicano dentro del ámbito comparado.

El presente estudio ilustra la implementación del artículo 50 del ADPIC en el sistema jurídico mexicano. Aquí resultan relevantes dos temas. El primero de ellos es que no se mencionó al artículo 50 del ADPIC en una sentencia que resolvió sobre la constitucionalidad de los preceptos jurídicos que lo implementan en el sistema jurídico mexicano.

El segundo tema tiene que ver con que en el sistema jurídico mexicano no existe un esquema de limitación a la responsabilidad de los proveedores de servicios de Internet, como los esquemas que existen en otros países desde hace alrededor de dieciocho años. Incluso, dicha limitante de responsabilidad ha sido incluida en los capítulos de propiedad intelectual de varios tratados de libre comercio celebrados por países de América Latina, posteriormente al TLCAN.

Si el artículo sección 97a del Copyright, Designs and Patents Act contiene un requisito extra, para el caso en que la medida vaya dirigida a proveedores de servicios de Internet, es porque dicho requisito viene dentro del esquema de limitación a la responsabilidad de los proveedores de servicios de Internet.

Dicho esquema no existe en el sistema jurídico mexicano probablemente debido a que los proveedores de servicios de Internet no se han visto en la necesidad de defender sus derechos frente a una acción en que, además de las medidas preliminares o definitivas como las analizadas en estos litigios, se les exija resarcimiento por daños y perjuicios. En este litigio, ese no fue el caso, no porque los proteja la legislación o algún precedente o decisión judicial. En este caso lo que protegió a los proveedores de servicios de Internet fue la mesura de la parte actora. De haber aplicado los supuestos jurídicos contenidos en las fracciones I y III del artículo 231 de la Ley Federal del Derecho de Autor a los proveedores de servicios de Internet, el resultado hubiera sido la declaración de infracción por parte 
de éstos. El siguiente paso hubiera sido pedir los daños y perjuicios ante la autoridad judicial. Dicho resultado sería así, sin importar que la exposición que tiene un proveedor de servicios de Internet a la responsabilidad por infracción a derechos de autor o derechos conexos sea originada de manera automatizada bajo las instrucciones y decisión de un suscriptor o de un tercero. 